\title{
Thermal-hydraulics/thermal-mechanics temporal coupling for unprotected loss of flow accidents simulations on a SFR
}

\author{
Cyril Patricot $^{1^{*}}$, Grzegorz Kepisty ${ }^{1}$, Karim Ammar $^{1}$, Guillaume Campioni ${ }^{1}$, and Edouard Hourcade ${ }^{2}$ \\ ${ }^{1}$ CEA, DEN, DM2S, SERMA, 91191 Gif-sur-Yvette, France \\ 2 CEA, DEN, DER, CPA, 13108 Saint-Paul-Lez-Durance Cedex, France
}

Received: 12 May 2015 / Accepted: 25 November 2015

Published online: 11 January 2016

\begin{abstract}
In the frame of ASTRID designing, unprotected loss of flow (ULOF) accidents are considered. As the reactor is not scrammed, power evolution is driven by neutronic feedbacks, among which Doppler effect, linked to fuel temperature, is prominent. Fuel temperature is calculated using thermal properties of fuel pins (we will focus on heat transfer coefficient between fuel pellet and cladding, $H_{\text {gap }}$, and on fuel thermal conductivity, $\left.\lambda_{\text {fuel }}\right)$ which vary with irradiation conditions (neutronic flux, mass flow and history for instance) and during transient (mainly because of dilatation of materials with temperature). In this paper, we propose an analysis of the impact of spatial variation and temporal evolution of thermal properties of fuel pins on a CFV-like core behavior during an ULOF accident. These effects are usually neglected under some a priori conservative assumptions. The vocation of our work is not to provide a best-estimate calculation of ULOF transient, but to discuss some of its physical aspects. To achieve this goal, we used TETAR, a thermal-hydraulics system code developed by our team to calculate ULOF transients, GERMINAL V1.5, a CEA code dedicated to SFR pin thermal-mechanics calculations and APOLLO ${ }^{\circledR}$, a neutronic code in development at CEA.
\end{abstract}

\section{Introduction}

The CFV (Cœur Faible Vidange, low void coefficient core) concept [1], which includes several innovations, is viewed as a way to improve the sodium void effect (reactivity effect of a core voiding) and the accidental behavior of large sodium fast reactors (SFRs). A scheme of this kind of core is given in Figure 1. A sodium plenum, with an upper absorbing protection, is positioned just above the core in order to increase the neutrons leakage in case of voiding. This effect is enhanced by the heterogeneities of the inner core, and by the height difference between the outer core and the inner core. These particularities increase the flux at the top of the core, and therefore in the plenum.

Loss of flow accidents are especially difficult for large SFRs and are therefore studied in depth in the frame of their designing. A detailed analysis of these accidents can be found in reference [2]. In order to clarify the explanations, our paper focuses on the unprotected loss of flow accident, during which primary pumps are lost, but not the secondary ones (we will call it $\mathrm{ULOF} / \mathrm{PP})$. The reactor is not scrammed, and the power evolution is driven by the neutronic feedbacks

\footnotetext{
* e-mail: cyril.patricot@cea.fr
}

(Doppler, sodium dilatation and dilatations of structures). During the accident, the coolant mass flow decreases until it reaches the natural convection equilibrium. It results in sodium heating in the upper part of the core, making the power decrease, thanks to CFV design. As a consequence, fuel temperature decreases and the Doppler effect is positive. Thus, the stabilization effect of the Doppler is, in this case, an obstacle to the power decrease.

An accurate evaluation of fuel temperature evolution during the transient is therefore necessary. It is usually derived from diffusion equation with given thermal properties. These properties are often homogenized over core zones and are usually constant in time. However, in reality, their spatial variations (mainly due to the heterogeneity of the core and to the mixing of sub-assemblies of different ages) and temporal evolutions (mainly due to differential thermal dilatations) can be quite important.

In this work, we propose an analysis of the impact of spatial variation and temporal evolution of thermal properties of fuel pins on a CFV-like core behavior during an ULOF/PP accident. Section 2 presents the evolution of the core under irradiation, calculated with APOLLO3 ${ }^{\circledR}$ [3] and GERMINAL V1.5 [4]. In Section 3, ULOF/PP accidents are calculated with TETAR (developed in the frame of TRIAD [5]) and different spatial descriptions of 


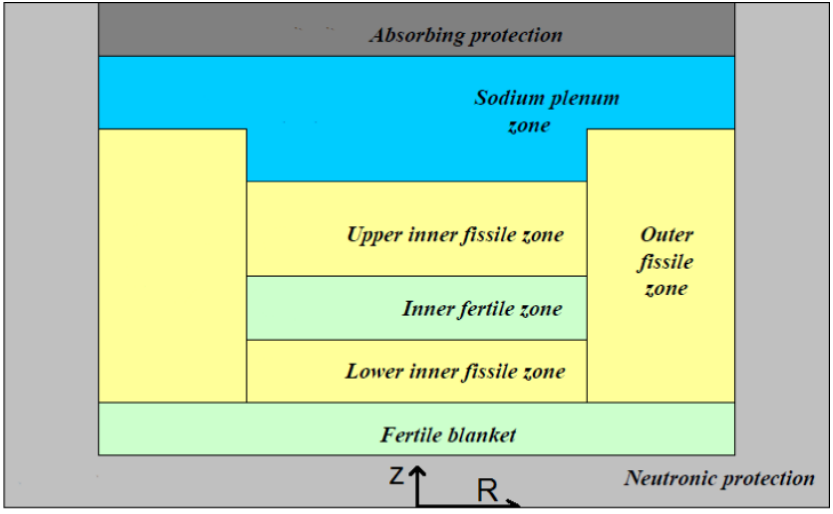

Fig. 1. Scheme of the CFV core concept.

thermal properties. In Section 4, we show the results of the temporal coupling. Section 5 provides some general conclusions.

Note that TETAR is not ASTRID reference tool and that the CFV-like core used is an academic model. As a consequence, the numerical results of this paper should not be considered as reference ones. They are given for the physical analysis of the phenomena.

\section{Core evolution under irradiation}

\subsection{Neutronic evolution}

We used APOLLO3 ${ }^{\circledR}$ for the neutronic calculations with 33 energy groups. Cross-sections were computed by the module ECCO of ERANOS [6]. Control rods are withdrawn in every calculation.

The chosen reloading procedure uses four batches. As the sub-assemblies are not moved during the reloading, the core is a mixing of sub-assemblies with different burn-up. The resulting power distribution is quite heterogeneous, as shown in Figures 2 and 3. In Figure 2, the power distribution is given, for a cut in the center of the core, at beginning of cycle.

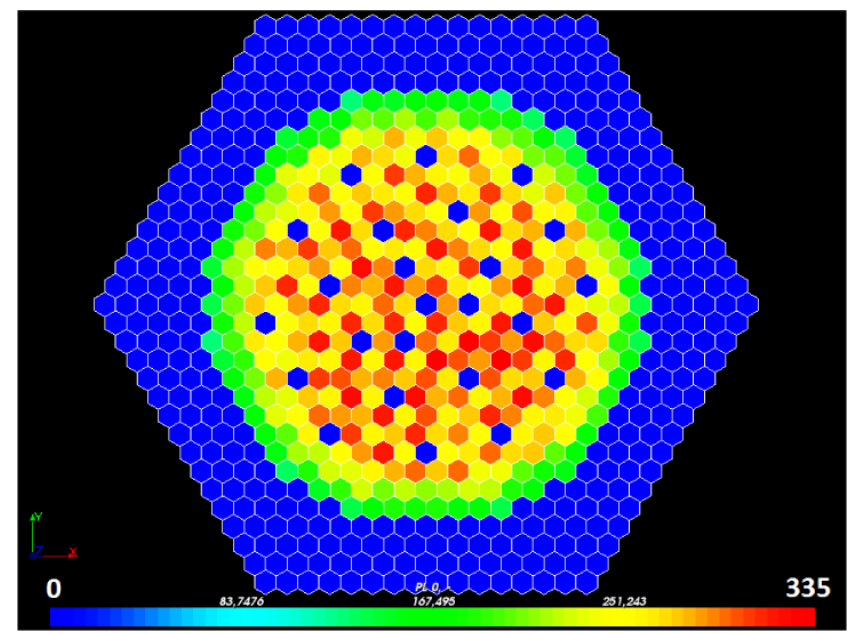

Fig. 2. Linear power distribution $(\mathrm{W} / \mathrm{cm}$ by pin) in the center of the core at beginning of cycle.

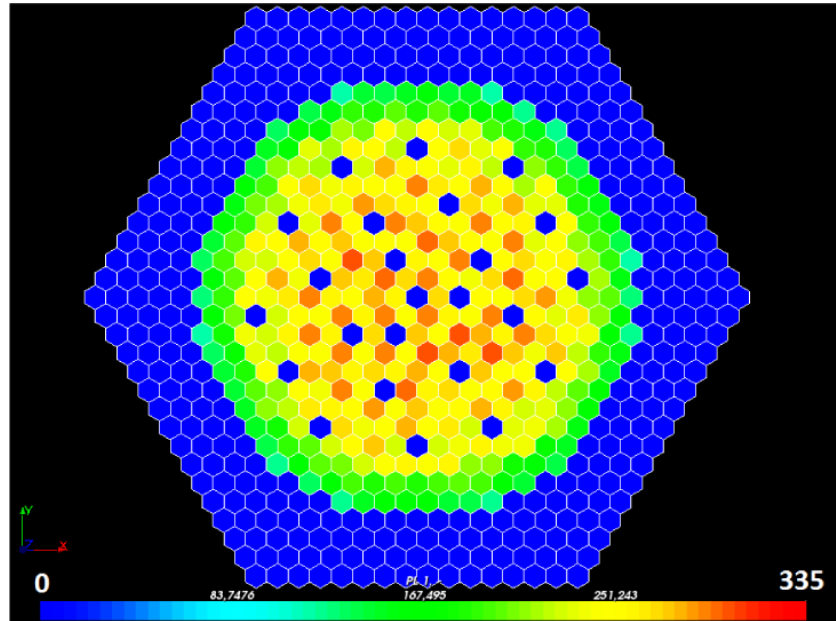

Fig. 3. Linear power distribution (W/cm by pin) in the center of the core at end of cycle.

Fresh sub-assemblies have high fissile content and have therefore a high linear power. At end of cycle, in Figure 3, the power distribution becomes more homogeneous. The color ranges are the same for both figures.

The same kind of flux and power redistributions occurs axially because of the combination of consumption of $\mathrm{Pu}$ in fissile zones and breeding in fertile ones (located at the bottom of the core).

\subsection{Thermo-mechanical evolution}

The evolution of thermo-mechanical properties of fuel pins is evaluated with GERMINAL V1.5. It uses simplify fuel description model based on mono-group neutron flux, linear power and irradiation damage distributions calculated by APOLLO ${ }^{\circledR}$. It also needs sodium inlet temperature and mass flow per pin.

The heat transfer coefficient between fuel pellet and cladding, called $H_{\text {gap }}$, has strong non-linear variations with irradiation. $H_{\text {gap }}$ and gap size evolutions are given in Figures 4 and 5 respectively, at a fixed position (in fissile) of

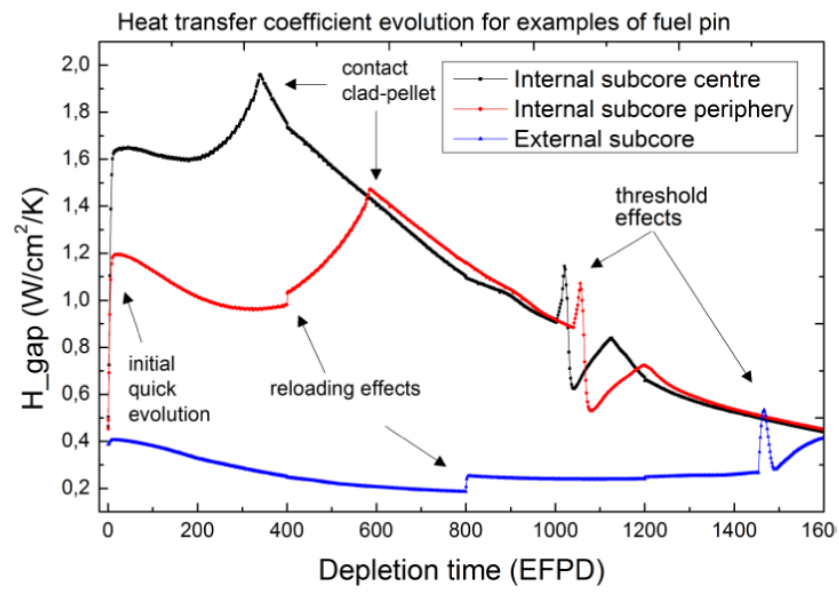

Fig. 4. Typical heat transfer coefficient evolution for chosen sub-assemblies (in top fissile zone). 


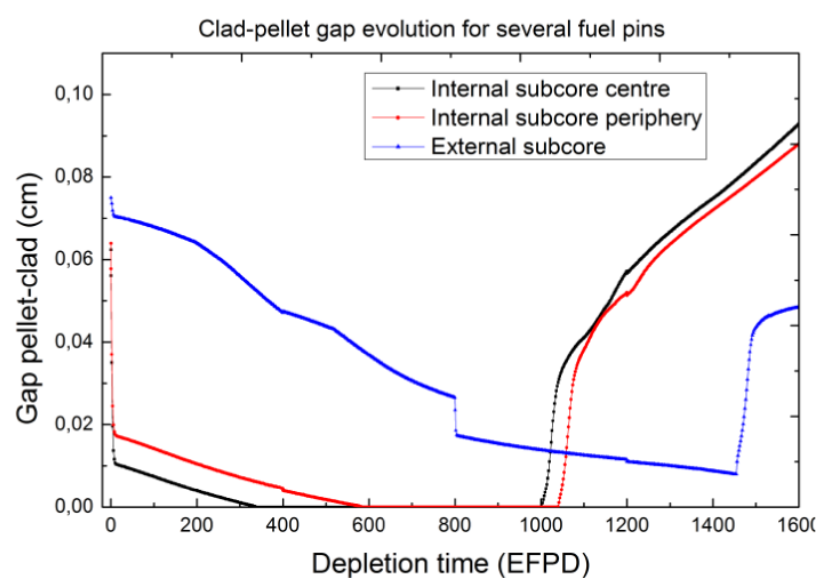

Fig. 5. Typical gap size evolution for chosen sub-assemblies (in top fissile zone).

chosen sub-assemblies. One can see that the initial thermal dilatation of the pellet makes the $H_{\text {gap }}$ increase, at the very beginning. A peak is then observed when the pellet comes in contact with the cladding (it does not occur here for the external subcore sub-assembly). A quite linear phase follows, with constant decrease of the $H_{\text {gap }}$ due to the degradation of the contact surface. Finally, threshold effects occur, swelling of the cladding, creation of an oxide layer on its surface and strong gaseous fission products release. The discontinuities at 400, 800 and $1200 \mathrm{EFPD}$ (equivalent full power days) are due to the reloading of a quarter of the core, which changes the linear power and flux in the studied sub-assemblies.

This non-linear behavior, together with the positioning of sub-assemblies in the core, and the axial heterogeneity of the fuel produce quite heterogeneous $3 \mathrm{D}$ maps of $H_{\text {gap }}$, as one can see in Figure 6. To build this 3D map, one mean pin per sub-assembly has been calculated. A 3D map of thermal conductivity of fuel (called $\lambda_{\text {fuel }}$ ) is given in Figure 7 . The evolution of this quantity is much more linear: the irradiation degrades the ceramics and thus its conductivity. As a consequence, $\lambda_{\text {fuel }}$ is maximal where the irradiation damages are minimal.

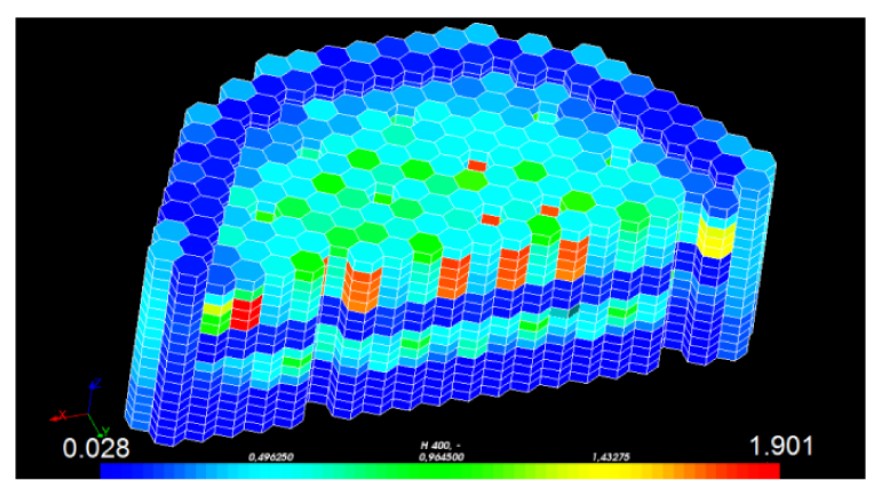

Fig. 6. Typical 3D map of $H_{g a p}\left(\mathrm{~W} / \mathrm{cm}^{2} / \mathrm{K}\right)$.

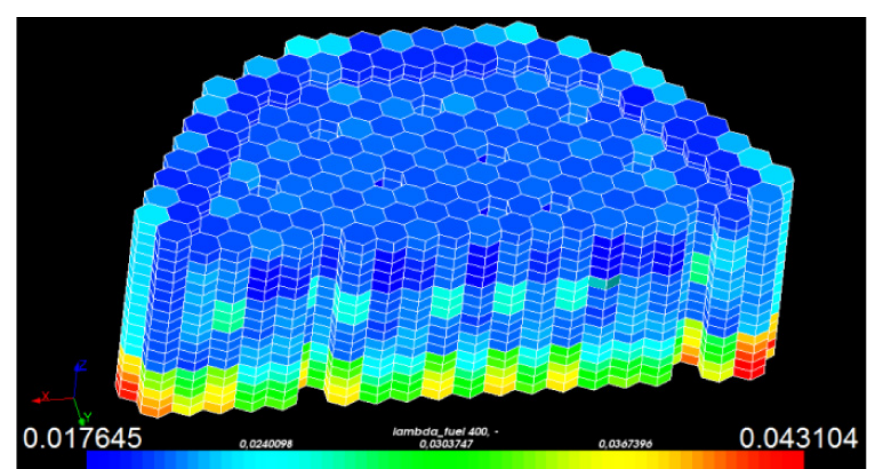

Fig. 7. Typical 3D map of $\lambda_{\text {fuel }}(\mathrm{W} / \mathrm{cm} / \mathrm{K})$.

\section{Impact of spatial descriptions of thermal properties and of neutronic feedbacks on the ULOF/PP accident}

\subsection{Calculations comparison with integrated neutronic feedback coefficients}

We used TETAR (Transients Estimation Tool for nAcooled Reactors) to calculate the ULOF/PP accident. It solves 1D thermo-hydraulic equations in each sub-assembly. We emphasize that each sub-assembly is calculated separately by a dedicated $1 \mathrm{D}$ thermo-hydraulic channel in all calculations presented in this paper. This ability of TETAR allowed us to perform our studies on spatial descriptions impacts. Mass flow in each sub-assembly is calculated to create a given pressure drop. Pin temperature is calculated through 1D diffusion. Point kinetic, fed with feedback coefficients (integrated or local) from APOL$\mathrm{LO} 3{ }^{\circledR}$, is used for the power estimation. The system is closed with sodium collectors and sodium-sodium heat exchangers simple models. The accident is driven by a given decrease of the pumps pressure. The overall pressure drop due to gravity (this term leads to natural convection) is calculated precisely.

In this section, the thermal properties are constant during the transient. Four models were used to estimate their initial value:

- Exact: one mean pin per sub-assembly is calculated by GERMINAL V1.5, and the results feed directly the TETAR calculation;

- Global average: we calculate, from the exact core calculation, the mean $H_{\text {gap }}$ and $\lambda_{\text {fuel }}$ of the core and use them everywhere in the TETAR calculation;

- Zones average: we calculate, from the exact core calculation, the mean $H_{g a p}$ and $\lambda_{\text {fuel }}$ of the core main five zones (Fig. 1). They are used in the corresponding meshes in TETAR;

- Groups: we gather sub-assemblies in groups and calculate one mean pin per group (sub-assemblies of the same ring, from the same batch). In comparison with the exact model, the number of GERMINAL V1.5 calculations is reduced by almost a factor 10 .

Sodium maximal temperature and power evolutions during the ULOF/PP accident are given in Figures 8 and 9 


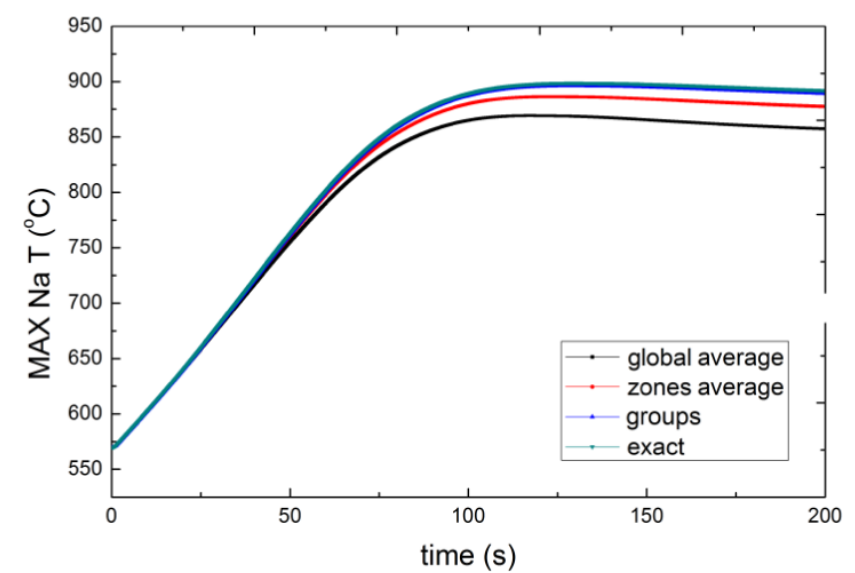

Fig. 8. Sodium maximal temperature during ULOF/PP accident for different thermal properties models.

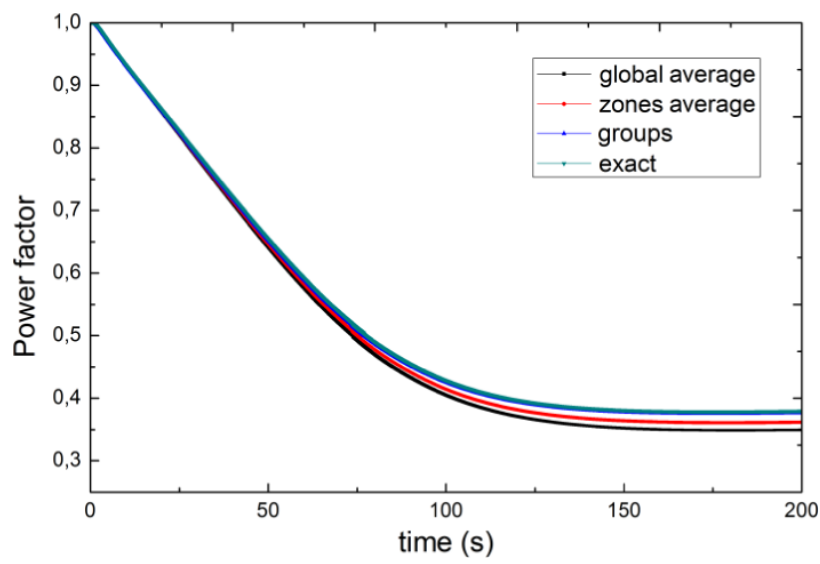

Fig. 9. Power during ULOF/PP accident for different thermal properties models.

for the models above. One can see that the exact and the group models are indistinguishable and that the maximal temperature they reached is slightly above the zone average model, which is slightly above the global average model.

Sodium maximal temperatures for some other models are given in Table 1. One can see that the zones average model is enough for $\lambda_{\text {fuel }}$, its results are very close to those of the exact model. In addition, non-linearities seem to be weak; the effect of a combination of models is the sum of the effects of the models. Finally, the difference between the groups and the exact models is very small in all cases, about $3{ }^{\circ} \mathrm{C}$.

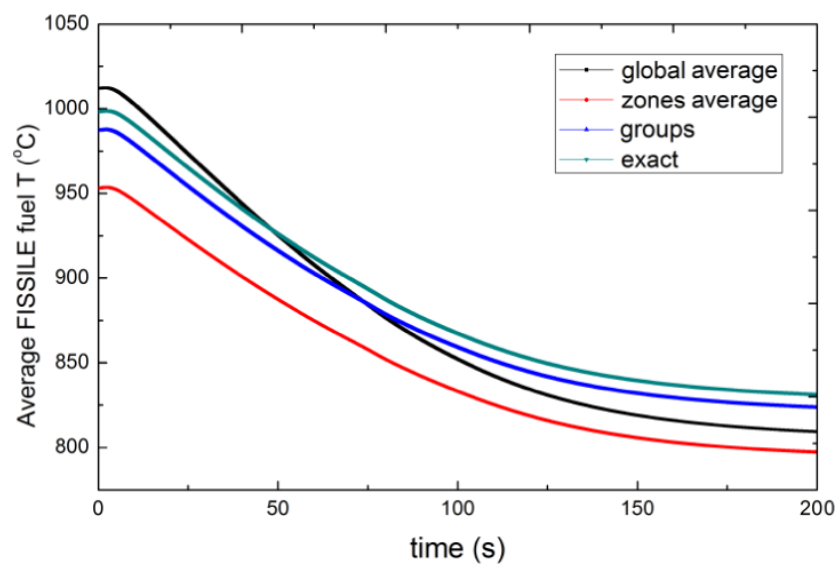

Fig. 10. Average fissile temperature during ULOF/PP for different $H_{\text {gap }}$ treatments.

\subsection{Interpretation of the results with integrated neutronic feedback coefficients}

All the presented calculations used two integrated Doppler coefficients, one for the fertile zones, and one for the fissile zones. The power is therefore affected by the average fissile and fertile temperatures. One can see their evolution in Figures 10 and 11, for the calculations of the second column of Table 1 ( $\lambda_{\text {fuel }}$ model is always global average). Except for the global average model which mixes fertile and fissile meshes, one can see that every $H_{\text {gap }}$ averaging leads to a cooler fuel.

This observation can be explained. Let us consider two fuel meshes, $i$ and $j$, in contact with the cladding. Because of the linearity of the diffusion equation, the temperature of $i$ can be written as:

$$
T_{i}=T_{i}^{C l}+\frac{\alpha_{i}}{h_{i}},
$$

with $T_{i}^{C l}$ the temperature of the cladding, $h_{i}$ the $H_{g a p}$ coefficient and $\alpha_{i}$ a scalar depending on local power. The same equation can be written for mesh $j$. We introduce now the temperatures $T_{i}^{m}$ and $T_{j}^{m}$ obtained using average $H_{\text {gap }}$ value, that is to say $\frac{h_{i}+h_{j} j}{2}$. The difference between the average values with exact and average $H_{g a p}$ is equal to:

$$
\frac{T_{i}^{m}+T_{j}^{m}}{2}-\frac{T_{i}+T_{j}}{2}=\frac{\left(h_{i}-h_{j}\right)\left(\frac{\alpha_{i}}{h_{i}}-\frac{\alpha_{j}}{h_{j}}\right)}{2\left(h_{i}+h_{j}\right)} .
$$

Table 1. Comparison of sodium maximal temperature $\left({ }^{\circ} \mathrm{C}\right)$ during ULOF $/ \mathrm{PP}$ accident for different thermal properties models.

\begin{tabular}{llll}
\hline Model & $H_{\text {gap }}\left(\lambda_{\text {fuel }}:\right.$ global average $)$ & $\lambda_{\text {fuel }}\left(H_{\text {gap }}:\right.$ global average $)$ & $H_{\text {gap }}$ and $\lambda_{\text {fuel }}$ \\
\hline Global average & 869.2 & 869.2 & 869.2 \\
Zones average & 877.4 & 877.8 & 886.4 \\
Groups & 887.2 & 878.3 & 896.1 \\
Exact & 889.9 & 878.6 & 898.9 \\
\hline
\end{tabular}




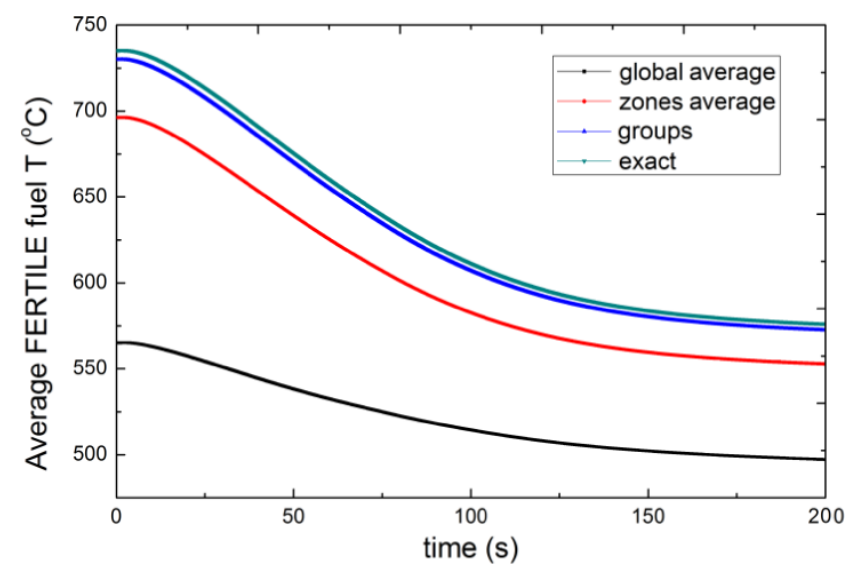

Fig. 11. Average fertile temperature during ULOF/PP for different $H_{\text {gap }}$ treatments.

$\frac{\alpha_{i}}{h_{i}}$ is the temperature increase between fuel and cladding. This equation means therefore that using average $H_{\text {gap }}$ reduces average fuel temperature if the $H_{\text {gap }}$ of the hottest mesh is smaller than the one of the coolest mesh. The point is that it may be the reason why the hottest mesh is the hottest. Therefore, without strong positive correlation between power and $H_{g a p}$, using average $H_{\text {gap }}$ usually reduces fuel temperature.

In addition, we can prove that, starting with a cooler fuel, for the same power decrease, the Doppler effect is smaller. To show that, let us write the temperature of a given mesh in the situation $i$ like:

$$
T_{i}=T^{C l}+\alpha_{i} P,
$$

with $T^{C l}$ the temperature of the cladding, $P$ the local power and $\alpha_{i}$ a scalar depending on mesh state. The same equation can be written for the same mesh in the situation $j$ by replacing $\alpha_{i}$ by $\alpha_{j}$.

Now we consider that the power becomes, at time $t, P . f$ with $f$ a given factor $(f<1$ in the case of a ULOF). The mesh contribution to the Doppler effect is:

$$
\frac{T_{i}(t)-T_{i}(t=0)}{T_{i}(t)} C=\frac{\alpha_{i} P(f-1)}{T^{C l}+\alpha_{i} P f} C,
$$

with $C$ a given feedback coefficient (usually $C<0$ ). Thanks to the form chosen for equation (4) (this is the usual one), the coefficient $C$ has no dependence on temperature. We assume here that the cladding temperature is constant. The difference between the Doppler contributions of the mesh in both situations is:

$$
\begin{gathered}
\frac{T_{i}(t)-T_{i}(t=0)}{T_{i}(t)} C-\frac{T_{j}(t)-T_{j}(t=0)}{T_{j}(t)} C \\
=\frac{T^{C l} P(f-1)\left(\alpha_{i}-\alpha_{j}\right) C}{\left(T^{C l}+\alpha_{i} P f\right)\left(T^{C l}+\alpha_{j} P f\right)} .
\end{gathered}
$$

This quantity is positive if $C<0, f<1$ and $\alpha_{i}>\alpha_{j}$. In other words, if the Doppler effect is negative and if the power decreases, we show that the Doppler effect is smaller for an initially cooler mesh. As a consequence, the power
Table 2. Comparisons of sodium maximal temperature $\left({ }^{\circ} \mathrm{C}\right)$ during ULOF $/ \mathrm{PP}$ accident with and without local neutronic feedbacks.

\begin{tabular}{lll}
\hline Model & $\begin{array}{l}H_{\text {gap }} \text { and } \lambda_{\text {fuel }} \\
\text { (and integrated NF) }\end{array}$ & $\begin{array}{l}H_{\text {gap }} \text { and } \lambda_{\text {fuel }} \\
\text { (and local NF) }\end{array}$ \\
\hline Global average & 869.2 & 878.9 \\
Zones average & 886.4 & 898.0 \\
Groups & 896.1 & 902.4 \\
Exact & 898.9 & 904.3 \\
\hline
\end{tabular}

decreases a little bit more. These results explain the impact of spatial description of thermal properties of fuel pins we observed in Section 3.1.

\subsection{Impact of local neutronic feedback coefficients}

The previous analysis is based on the use of average fuel temperatures to calculate the Doppler feedback. One could wonder if it still stands if we use local neutronic feedbacks. Because this work is on the impact of the fuel pin thermomechanics on ULOF/PP accident, we focused our study on the Doppler effect. Comparisons of sodium maximal temperatures reached during $\mathrm{ULOF} / \mathrm{PP}$ accident with and without local Doppler coefficients (the global Doppler effect is the same) are presented in Table 2. 3D maps of Doppler coefficients, derived from the perturbation theory, are given in Figure 12 (fissile) and Figure 13 (fertile).

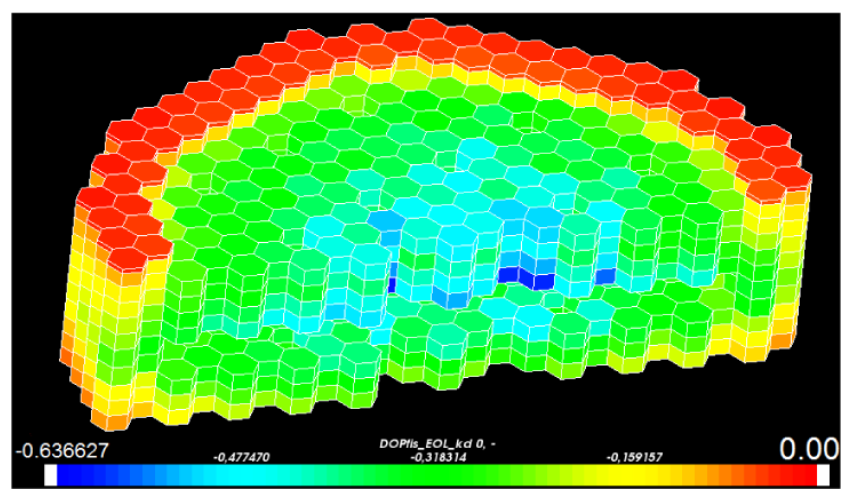

Fig. 12. 3D map of Doppler coefficients (pcm) in fissile zones.

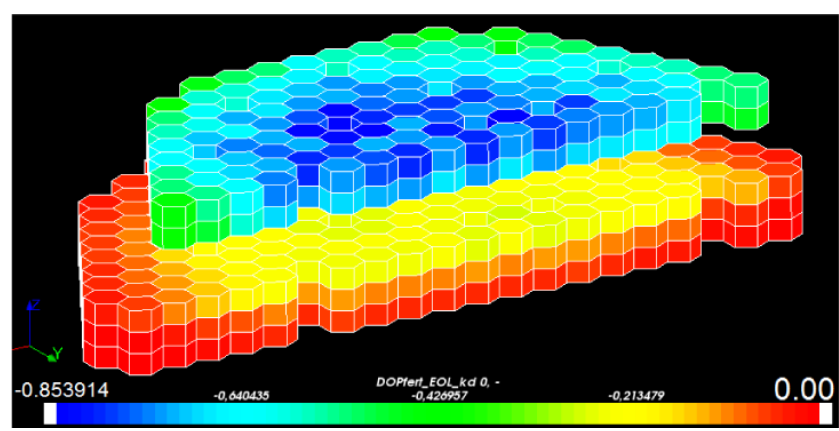

Fig. 13. 3D map of Doppler coefficients (pcm) in fertile zones. 
One can see that, here, using local neutronic feedbacks always increases the sodium maximal temperature. The impact depends on the thermal properties model, but is pretty small (about $5^{\circ} \mathrm{C}$ ) for exact treatment of $H_{\text {gap }}$ and $\lambda_{\text {fuel }}$. An analysis shows that this difference is mainly due to the heterogeneity of the fertile zones. Indeed, in the inner fertile there are in the same time a much stronger fuel temperature decrease and much stronger Doppler coefficients than in the fertile blanket (this is visible in Fig. 13). These two differences together create a bias when one uses integrated Doppler coefficients.

In Section 3, we saw the impact of spatial description of thermal properties. The more accurate it is, the hotter the sodium becomes during the ULOF/PP accident, whatever is the spatial treatment of the Doppler effect. We will now see the impact of the temporal evolution of the thermal properties.

\section{Impact of temporal evolution of thermal properties during the ULOF/PP accident}

\subsection{Calculations comparison with integrated neutronic feedback coefficients}

We used the simple explicit coupling scheme illustrated in Figure 14. GERMINAL V1.5 gives local $H_{\text {gap }}$ and $\lambda_{\text {fuel }}$ values to TETAR, which returns mass flow per pin and local power (through the global power factor calculated by the point kinetic). The coupling time step is set to $10 \mathrm{~s}$. The already presented groups model for GERMINAL V1.5 is chosen in order to save calculation time.

It would be interesting to enhance this coupling scheme, and it should be done in future work. However, preliminary studies show that this scheme is correct.

The results of the coupled calculation are given in Figures 15 and 16 with equivalent non-coupled case. Here, integrated Doppler coefficients are used. One can see that the temporal coupling has a very strong impact, about $-38^{\circ} \mathrm{C}$.

\subsection{Interpretation of the results with integrated neutronic feedback coefficients}

This very strong impact of the coupling is due to the opening of the gap during the transient: as the cladding is

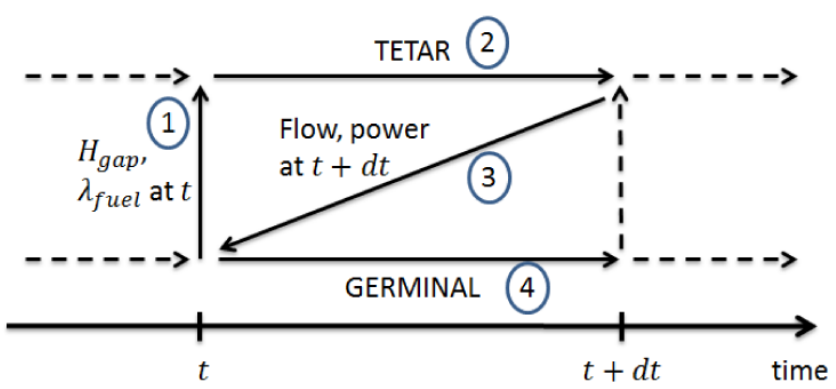

Fig. 14. The temporal coupling scheme used.

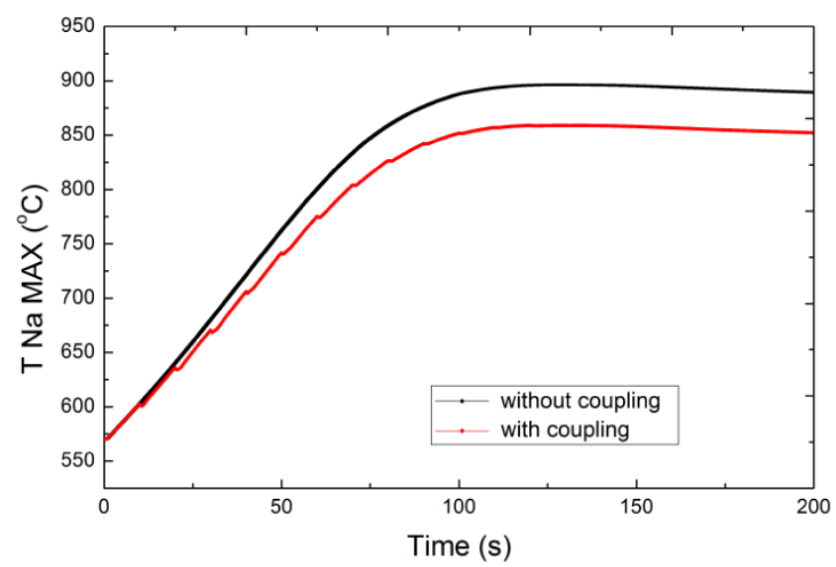

Fig. 15. Sodium maximal temperature during ULOF/PP accident with and without coupling.

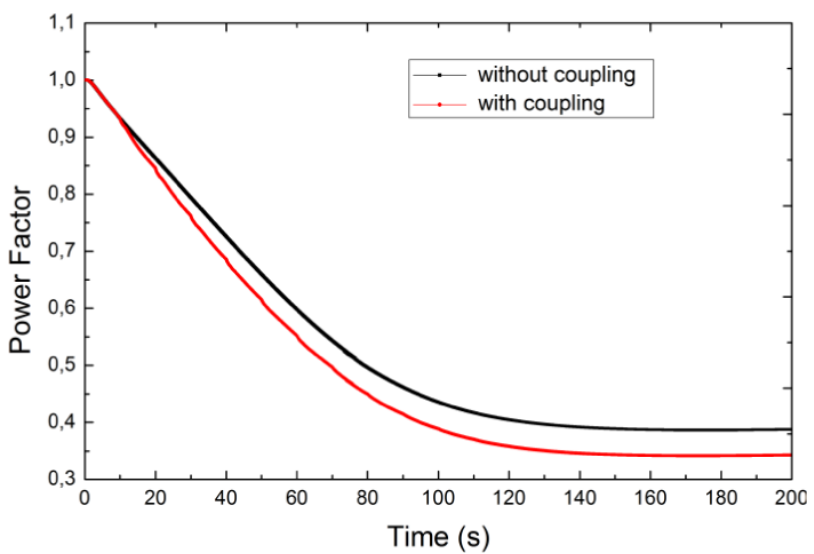

Fig. 16. Power during ULOF/PP accident with and without coupling.

getting hotter and the fuel cooler, the differential thermal expansion takes them away from each other. As a consequence, the $H_{\text {gap }}$ decreases and the fuel temperature drop is reduced, leading to a smaller Doppler effect.

$H_{\text {gap }}$ profile evolutions for one sub-assembly from the inner core is given in Figure 17, and for one sub-assembly from the outer core in Figure 18. The $H_{\text {gap }}$ does decrease everywhere. It is especially important (divided by about 3 ) where the initial value was high: there was a contact between the cladding and the pellet at these locations. This contact is lost during the transient.

While $H_{\text {gap }}$ changes a lot during the transient, $\lambda_{\text {fuel }}$ is found to be almost constant.

One can note that the $H_{\text {gap }}$ temporal evolution is rather smooth, and could be approximated by polynomial functions, as suggested in reference [2].

\subsection{Impact of local neutronic feedback coefficients}

We found, in Section 3, that local neutronic feedback coefficients have a small impact on ULOF/PP when used with a good spatial discretization of the thermal properties 


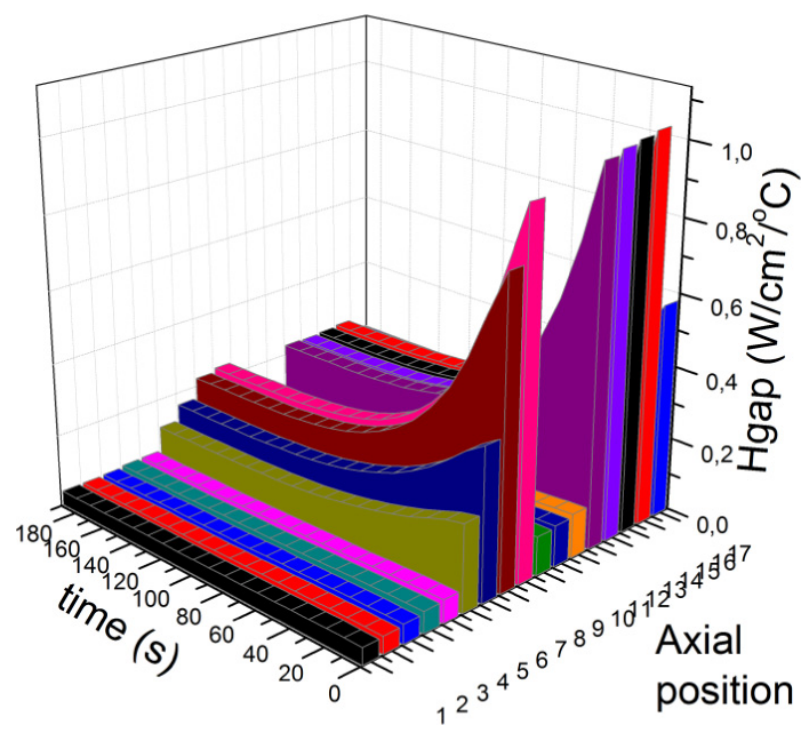

Fig. 17. $H_{\text {gap }}$ profile evolutions during ULOF/PP accident for one sub-assembly from the inner core.

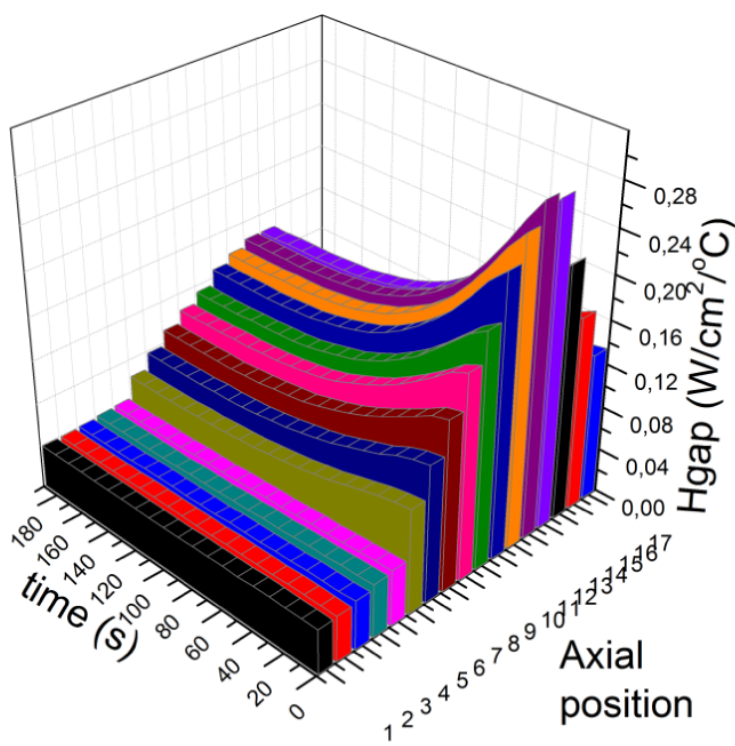

Fig. 18. $H_{\text {gap }}$ profile evolutions during ULOF/PP accident for one sub-assembly from the outer core.

of the pins (see Tab. 2). The cause of the discrepancy has been identified to be the combined heterogeneities of $H_{\text {aap }}$ and Doppler coefficients in the fuel zones. The temporal coupling, because it reduces the $H_{\text {gap }}$ preferentially where it is high, that is to say in the center of the core, where the Doppler effect is the strongest, reduces these heterogeneities. As a consequence, the impact of the local feedback coefficients is reduced. This is visible in Figure 19. We used, here again, the groups model for $H_{\text {gap }}$ and $\lambda_{\text {fuel }}$.

The comparison with the non-coupled equivalent calculation with local feedbacks coefficients leads to a reduction of the sodium maximal temperature of about $45^{\circ} \mathrm{C}$.

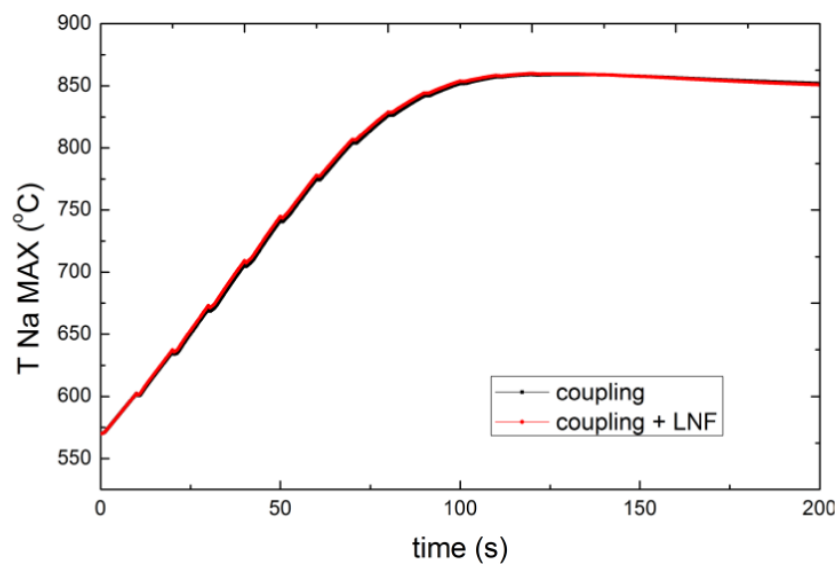

Fig. 19. Sodium maximal temperature during ULOF/PP accident with coupling and different Doppler effect treatments.

\section{Conclusions}

In this paper, we proposed an analysis of the impact of spatial variation and temporal evolution of thermal properties of fuel pins on the behavior of a CFV-like core during an ULOF accident.

Sources of spatial variations and temporal evolution of the main thermal properties of fuel pins were identified. The impact of their spatial variations was found to be about $+30{ }^{\circ} \mathrm{C}$ on sodium temperature during ULOF/PP transient. It is mainly due to $H_{\text {gap }}$, and simple zones averages seem to be enough for $\lambda_{f u e l}$. The combined effect of local thermal properties and local Doppler coefficients leads to an impact of about $+35^{\circ} \mathrm{C}$. On the other hand, the temporal coupling, because of the opening of the gap, improves the reactor behavior during the $\mathrm{ULOF} / \mathrm{PP}$ and leads to a decrease of about $45^{\circ} \mathrm{C}$ of the sodium temperature. This improvement of the core behavior is very strong and could help greatly to demonstrate the safety of large SFRs.

From the above observations we can make the following comments:

- a static estimation of $\lambda_{\text {fuel }}$ in the main zones of the core is sufficient;

- for a conservative calculation, the spatial variations of $H_{\text {gap }}$ and of the Doppler effect should be taken into account;

- the temporal coupling between thermal-hydraulics and thermal-mechanics of fuel pins brings out substantial margins, because of the $H_{\text {gap }}$ evolution.

\section{References}

1. M.S. Chenaud et al., Status of the ASTRID core at the end of the pre-conceptual design phase 1 , in Proceedings of ICAPP 2013, Jeju Island, Korea, 2013 (2013)

2. R. Lavastre et al., State of art of CATHARE model for transient safety analysis of ASTRID SFR, in Proceedings of NUTHOS-10, Okinawa, Japan, 2014 (2014)

3. H. Golfier et al., APOLLO3: a common project of CEA, AREVA and EDF for the development of a new deterministic 
multi-purpose code for core physics analysis, in Proceedings of MEC 2009, New York, USA, 2009 (2009)

4. L. Roche, M. Pelletier, Modelling of the thermomechanical and physical process in FR fuel pins using GERMINAL code, in Proceedings of MOX Fuel Cycle Technologies for Medium and Long Term Deployment IAEA-SM-358/25, Vienna, Austria, 1999 (1999), pp. 322-335
5. E. Hourcade et al., SFR core design: a system-driven multicriteria core optimisation exercise with TRIAD, in Proceedings of FR13, Paris, France, 2013 (2013)

6. G. Rimpault et al., The ERANOS code and data system for fast reactor neutronic analyses, in Proceedings of PHYSOR 2002, Seoul, Korea, 2002 (2002)

Cite this article as: Cyril Patricot, Grzegorz Kepisty, Karim Ammar, Guillaume Campioni, Edouard Hourcade, Thermalhydraulics/thermal-mechanics temporal coupling for unprotected loss of flow accidents simulations on a SFR, EPJ Nuclear Sci.

Technol. 2, 2 (2016) 